\title{
Obtención de espumas metálicas utilizando como metal principal aluminio comercial y reciclado con diversos agentes espumantes
}

\section{Obtaining metallic foams using commercial and recycled aluminum as the main metal with various foaming agents}

Presentación: 01/12/2021

Aprobación: 04/02/2022

\section{José M. Espasandin}

Grupo de Investigación de Materiales (GIM)-Facultad Regional Resistencia, Universidad Tecnológica Nacional-Argentina josemanuelespasandin@gmail.com

\section{Ofelia J.S. Canteros}

Grupo de Investigación de Materiales (GIM)- Facultad Regional Resistencia, Universidad Tecnológica Nacional-Argentina jessicanteros@gmail.com

\section{Leonardo G. Barabas}

Grupo de Investigación de Materiales (GIM)- Facultad Regional Resistencia, Universidad Tecnológica Nacional-Argentina leonardogbarabas@hotmail.com

\section{María T. Malachevsky}

Centro Atómico Bariloche-Argentina

malachevsky@yahoo.com

\section{Resumen}

Se realizó el presente trabajo basado en la fabricación de espumas metálicas mediante la técnica de pulvimetalurgia. Para la fabricación de muestras, se emplearon polvos de aluminio comercial y reciclado. En algunos experimentos se adicionó cobre en polvo como aleante, tanto comercial como reciclado. Se utilizaron bicarbonato de sodio $\left(\mathrm{NaHCO}_{3}\right)$ y 
carbonato de calcio $\left(\mathrm{CaCO}_{3}\right)$ cómo agentes espumantes. Los polvos se mezclaron en diferentes proporciones. Se sometieron a un prensado con presiones y temperaturas diversas. Se espumaron en el horno a temperaturas de entre $640{ }^{\circ} \mathrm{C}$ y $900{ }^{\circ} \mathrm{C}$ en periodos de tiempos que varían entre 1,5 minutos y 30 minutos. Todos los métodos y procedimientos utilizados permitieron la obtención de espumas, que mediante imágenes fotográficas y/o tomografía de rayos $\mathrm{X}$ demuestran cualitativamente diferencias estructurales. Tales como el tipo, forma y distribución de los poros, y aparición de fallas internas (fisuras) normales a la dirección de compactación de la muestra durante el proceso de compresión unidireccional de los polvos en el estado verde.

Palabras claves: aluminio, bicarbonato de sodio $\left(\mathrm{NaHCO}_{3}\right)$, carbonato de calcio $\left(\mathrm{CaCO}_{3}\right)$, Cobre (Cu), pulvimetalurgia.

\begin{abstract}
:
The present work was based on the manufacture of metal foams using the powder metallurgy technique. For the production of samples, commercial and recycled aluminium powders were used. In some experiments, powdered copper was added as an allele, both commercial and recycled. Sodium bicarbonate $\left(\mathrm{NaHCO}_{3}\right)$ and calcium carbonate $\left(\mathrm{CaCO}_{3}\right)$ were used as foaming agents. The powders were mixed in different proportions. They were pressed with varying pressures and temperatures. They were foamed in the oven at temperatures between $640{ }^{\circ} \mathrm{C}$ and $900{ }^{\circ} \mathrm{C}$ in periods ranging from 1.5 minutes to 30 minutes. All the methods and procedures used made it possible to obtain foams, which by means of photographic images and/or X-ray tomography demonstrate qualitative structural differences. Such as the type, shape and distribution of pores, and the appearance of internal faults (cracks) normal to the compaction direction of the sample during the process of unidirectional compression of powders in the green state.
\end{abstract}

Key words: aluminium, sodium bicarbonate $\left(\mathrm{NaHCO}_{3}\right)$, calcium carbonate $\left(\mathrm{CaCO}_{3}\right)$, Copper $(\mathrm{Cu})$, powder metallurgy.

\title{
1. Introducción
}

La utilización de los metales celulares en la industria está creciendo debido a sus propiedades físicas y mecánicas, principalmente a su bajo peso brindado por su estructura porosa de baja densidad. Sus mayores aplicaciones se dan en la absorción de energía de impacto en el sector automotriz, intercambiadores de calor, filtros, paneles sándwich en la construcción, entre otros. Sin embargo, su uso está limitado por su alto costo, debido a la necesidad de utilizar tecnologías y agentes espumantes como el Hidruro de Titanio que encarece su producción. En este sentido, nuestro esfuerzo se focalizó en la reutilización de materiales, reciclando aluminio para la obtención de espumas y utilizando agentes espumantes alternativos.

Originalmente el hidruro de titanio $\left(\mathrm{TiH}_{2}\right)$ se aplicó principalmente como uno de los agentes espumantes más atrayentes tanto para la fundición como para los procesos por pulvimetalurgia (Fernández et al., 2009) en la obtención de espumas de aluminio y aleaciones del mismo. El papel del agente espumante, sin importar cuál sea el proceso de fabricación de la espuma, es liberar gas, puesto que el metal deberá encontrarse 
en estado líquido o semilíquido dependiendo de las características de la aleación. Sin embargo, como lo discutieron en detalle Gergely, Currand y Clyne (Gergely et al., 2003; Geramipour et al., 2017) existen ciertos factores que están involucrados en la selección del agente espumante para la formación de las espumas. Estos incluyen: (I) la cinética y las características termodinámicas de reacciones de descomposición y reacciones entre las partículas del agente espumante y aleación fundida o en estado intermedio; (II) la influencia de productos de descomposición y gas espumoso en la estabilización de espumas; y (III), para nosotros más allá de que no es una influencia técnica, si lo es el alto costo económico (Titanium (II) hydride, s. f.) lo que hace difícil la disponibilidad del hidruro de titanio $\left(\mathrm{TiH}_{2}\right)$ como agente espumante principal.

Entre las limitaciones que posee el hidruro de titanio $\left(\mathrm{TiH}_{2}\right)$ podemos enumerar, que tiene un alto costo económico (Titanium (II) hydride, s. f.) acorde a lo descripto en el párrafo precedente, su temperatura de descomposición es baja comparada con las temperaturas de fundición del aluminio y de las temperaturas de estados intermedios de aleaciones de aluminio/cobre en su zonas de transformación hipo e hipereutécticas, para elevar la temperatura de espumado debemos tratarlo térmicamente, solo actúa como agente de expansión y no favorece la estabilidad de la espuma, y su densidad es superior a la del metal a espumar ocasionando la no uniformidad en la espuma.

La propuesta es buscar otras alternativas de agentes espumantes entre los cuales aparecen como opción el carbonato de calcio $\left(\mathrm{CaCO}_{3}\right)($ Calcium carbonate, s. f.) y el bicarbonato de sodio $\left(\mathrm{NaHCO}_{3}\right)$ (Sodium bicarbonate, s. f.).Ambos son de muy bajo costo comparado con el hidruro de titanio con densidades más cercanas a las del aluminio y las aleaciones estudiadas en el presente (Kevorkijan et al., 2011).

La descomposición del $\mathrm{CaCO}_{3}$ se produce entre los $600{ }^{\circ} \mathrm{C}$ y $900{ }^{\circ} \mathrm{C}$ y el bicarbonato de sodio lo hace entre los $300^{\circ} \mathrm{C}$ y $360^{\circ} \mathrm{C}$, todo ello dependiendo del tamaño de partícula y de las condiciones físicas en las que se encuentran.

En algunas patentes tempranas (Nakamura et al., 2002) y en el trabajo reciente de Nakamura y colaboradores (Byakova, Nakamura et al., 2017) se encontró que el uso de $\mathrm{CaCO}_{3}$ es potencialmente adecuado como agente espumante para la obtención de espumas mediante el método de fundición del metal base. Además, Bryat (Bryat et al.; 2008) informó que el $\mathrm{CaCO}_{3}$ actúa como agente espumante cuando se encuentra en contacto con el aluminio en estado líquido y, al mismo tiempo, a través de los productos de descomposición tiene un efecto sobre la estabilidad de la espuma, permitiendo la formación de "espumas de aluminio autoestabilizadas".

Aunque la aplicación de $\mathrm{CaCO}_{3}$ como agente espumante en forma directa e indirecta en el espumado de aleaciones y compuestos de aluminio, ya se investigó. Existe la necesidad de un estudio adicional de la influencia del $\mathrm{CaCO}_{3}$ para la obtención de espumas por los métodos de pulvimetalurgia, y no solo por los tradicionales de fundición, debiendo verificarse el comportamiento de las reacciones y los resultados obtenidos.

Con el espíritu de trabajar en la búsqueda de alternativas para la obtención de un material novedoso con muchas aplicaciones en la industria, se realizó la comparación de espumas obtenidas con polvo de aluminio comercial y reciclado como metal base. Algunas de estas muestras fueron sometidas a estudio mediante tomografía de rayos $\mathrm{x}$ por lo cual se logró determinar visualmente la homogeneidad en la porosidad, el tipo de porosidad y defectos internos. 


\section{Desarrollo experimental}

\subsection{Polvos empleados}

Se utilizó como elemento base el aluminio y cómo aleante el cobre, ambos en forma de polvo comercial y obtenidos mecánicamente. El método mecánico de obtención de los polvos se da mediante un proceso de mecanizado con herramienta manual partiendo del material en bloque.

Para determinar el grado de pureza de estos polvos precursores se realizó un análisis de difracción de rayos $\mathrm{X}$ a cada una de estas muestras en un equipo PANalytical Empyrean, utilizando radiación de $\mathrm{CuK}_{\alpha}$, una tensión de $40 \mathrm{kV}$, una intensidad de $30 \mathrm{~mA}$ y un paso de $0.026^{\circ}$ cada $2 \mathrm{~s}$. Este equipo pertenece a la Comisión Nacional de Energía Atómica y se encuentra en el Centro Atómico de la Ciudad de Bariloche, Provincia de Rio Negro, República Argentina.

\subsection{Proceso para la obtención de espumas}

Las espumas metálicas que se obtuvieron por pulvimetalurgia se realizaron a partir de polvos precursores de metales o aleación base. Se sometieron a un proceso de mezclado manual o mecánico en conjunto con el compuesto espumante. La mezcla manual se realizó con mortero y la mecánica con molino de bolas.

Se procedió con el prensado en frío de la mezcla de polvos en matrices de entre 15 $\mathrm{mm}$ y $17 \mathrm{~mm}$ de diámetro, con presiones que oscilaron desde $40 \mathrm{MPa}$ a $220 \mathrm{MPa}$ por tiempos definidos. De manera continua se procedió al incremento de la temperatura para el prensado en caliente, con rangos de $150{ }^{\circ} \mathrm{C}$ a $600{ }^{\circ} \mathrm{C}$ y presiones de $150 \mathrm{MPa}$ a $450 \mathrm{MPa}$.

Las muestras se espumaron sometiendo las mismas a un salto térmico dentro de un horno precalentado a temperaturas de $700{ }^{\circ} \mathrm{C}$ a $900{ }^{\circ} \mathrm{C}$. Se evaluaron tiempos de permanencia dentro del mismo para lograr la optimización de las espumas.

\subsection{Preparación de muestras}

2.3.1. Espumas con polvo de aluminio comercial, polvo de cobre como aleante y $\mathrm{CaCO}_{3}$ cómo agente espumante.

Para la fabricación de espumas de aluminio y aleaciones del mismo, se partió de polvo comercial cómo metal base. El compuesto utilizado para el espumado de las muestras fue el carbonato de calcio $\left(\mathrm{CaCO}_{3}\right)$. El $70 \%$ de las pruebas fueron solo con aluminio y compuesto espumante y en el $30 \%$ se utilizó como elemento aleante polvo de cobre en porcentajes que se especificarán más adelante.

La obtención de las muestras con aluminio y compuesto espumante $\left(\mathrm{CaCO}_{3}\right)$ se efectuó con polvo comercial del metal. El polvo de aluminio se trató mediante molienda manual por un periodo de 10 minutos con la finalidad de disminuir el tamaño de partículas sin evaluar posteriormente el resultado del proceso. Ese polvo precursor se utilizó para la elaboración de dos muestras (A1 y A2). Tres de las muestras se elaboraron ejecutando la molienda del polvo en un molino de bolas, con esferas de acero al cromo, por un periodo de 1 hora con una velocidad de rotación de $18 \mathrm{rpm}$ (A3, A4 y A5). Las muestras restantes (A12 y A13) fueron mezcladas con polvo de cobre comercial y obtenido mediante trabajo de mecanizado. Se molieron en conjunto el polvo de aluminio con el de cobre manualmente por un plazo de 10 minutos. 
Procesado mecánicamente el polvo de aluminio solo y este mismo con cobre, se procedió a la mezcla con el agente espumante $\left(\mathrm{CaCO}_{3}\right)$. Todas las mezclas con el compuesto espumante se realizaron manualmente con mortero cerámico.

Se efectuó un prensado en frío de entre uno y hasta 5 minutos ejerciendo presiones en los rangos que van desde los $43 \mathrm{MPa}$ y $216 \mathrm{MPa}$ según la muestra. Se elevó la temperatura de la muestra dentro de la matriz entre los $450{ }^{\circ} \mathrm{C}$ y $580{ }^{\circ} \mathrm{C}$ elevando la presión entre $199 \mathrm{MPa}$ y $432 \mathrm{MPa}$ manteniendo estas condiciones por tiempos de $10 \mathrm{a}$ 30 minutos.

La etapa de espumado se efectuó con la introducción de las muestras en su estado verde en el horno a temperaturas que variaron de $720^{\circ} \mathrm{C}$ a $900^{\circ} \mathrm{C}$, y tiempos propuestos de expansión de 5 a 30 minutos.

2.3.2 Espumas con polvo de aluminio reciclado procesado mecánicamente, polvo de cobre como aleante y cómo agentes espumantes $\mathrm{CaCO}_{3}$ y $\mathrm{NaHCO}_{3}$.

En este caso se empleó aluminio reciclado por el cual se obtuvo polvo mediante un proceso de mecanizado manual, se incorporó a la mezcla nuevamente polvo de $\mathrm{Cu}$ cómo aleante. La obtención de las muestras de espumas de aluminio se realizó utilizando dos compuestos espumantes, $\mathrm{CaCO}_{3}$ y $\mathrm{NaHCO}_{3}$.

$\mathrm{El} \mathrm{CaCO}_{3}$ se adiciono en un $15 \%$ en peso del total de la muestra. La mezcla de polvos se trató mediante molienda manual por un periodo de 10 minutos con la finalidad de homogeneizar la dispersión de los polvos. El mismo se utilizó para la elaboración de una muestra (A7). Se efectuó un prensado en frío de 10 minutos ejerciendo una presión de $194 \mathrm{MPa}$. Se elevó la temperatura de la muestra dentro de la matriz hasta los $300{ }^{\circ} \mathrm{C}$ manteniendo la presión por 15 minutos más, proceso mediante el cual se obtiene la muestra en un estado llamado verde previo al espumado. La muestra, en dicho estado, se introduce en el horno previamente calentado a una temperatura de $640{ }^{\circ} \mathrm{C}$ considerando un tiempo de expansión de aproximadamente 7 minutos.

El bicarbonato de sodio $\left(\mathrm{NaHCO}_{3}\right)$, se utilizó como segundo agente espumante mediante el cual se obtuvieron dos muestras (A8 y A9). La relación de porcentajes de metal base, metal aleante y agente espumante se mantuvo para ambas muestras. El porcentaje en peso del agente espumante fue del $15 \%$, trabajándose la muestra mediante mezcla mecánica por un periodo de 20 minutos. Se procedió al prensado en frío durante 10 minutos con una presión de $194 \mathrm{MPa}$. Se elevó la temperatura a $150{ }^{\circ} \mathrm{C}$ a presión constante durante 20 minutos con lo que se concluyó el prensado en caliente. En la etapa de espumado es donde se diferenció la muestra A8 de la A9. Diferencia está, que radica en el tiempo de espumado dentro del horno que fue de 2 y 1,5 minutos respectivamente a la misma temperatura de $900{ }^{\circ} \mathrm{C}$.

Cómo resumen de lo expuesto en los puntos 2.2 y 2.3 se presenta a continuación la Tabla $\mathrm{N}^{\circ} 1$, donde pueden observarse los parámetros más relevantes. 


\begin{tabular}{|c|c|c|c|c|c|c|c|c|c|c|}
\hline \multirow[b]{2}{*}{ Muestra } & \multirow{2}{*}{$\begin{array}{c}\text { MEZCLA } \\
\text { Metal Base/ } \\
\text { Espumante/ } \\
\text { Aditivo }\end{array}$} & \multicolumn{2}{|c|}{ MOLIENDA } & \multicolumn{2}{|c|}{$\begin{array}{l}\text { PRENSADO } \\
\text { EN FRÍO }\end{array}$} & \multicolumn{3}{|c|}{$\begin{array}{l}\text { PRENSADO EN } \\
\text { CALIENTE }\end{array}$} & \multicolumn{2}{|c|}{ HORNO } \\
\hline & & $\stackrel{\mathrm{n}}{(\mathrm{rpm})}$ & $\stackrel{\mathrm{t}}{\mathrm{min}}$ & $\stackrel{\mathrm{t}}{\mathrm{min})}$ & $\begin{array}{c}\mathrm{P} \\
(\mathrm{MPa})\end{array}$ & $\begin{array}{c}\mathrm{t} \\
(\mathrm{min})\end{array}$ & $\begin{array}{c}\mathrm{P} \\
(\mathrm{MPa})\end{array}$ & $\begin{array}{c}\mathrm{T} \\
\left({ }^{\circ} \mathrm{C}\right)\end{array}$ & $\begin{array}{c}\mathrm{T} \\
\left({ }^{\circ} \mathrm{C}\right)\end{array}$ & $\stackrel{t}{t}$ \\
\hline A1 & $\mathrm{Al} / \mathrm{CaCO}_{3}$ & M & 10 & 1 & 43 & 30 & 198 & 450 & 800 & 30 \\
\hline A2 & $\mathrm{Al} / \mathrm{CaCO}_{3}$ & M & 10 & 1 & 43 & 15 & 198 & 450 & 900 & 30 \\
\hline A3 & $\mathrm{Al} / \mathrm{CaCO}_{3}$ & 18,8 & 60 & 5 & 216 & 15 & 432 & 450 & 900 & 30 \\
\hline A4 & $\mathrm{Al} / \mathrm{CaCO}_{3}$ & 18,8 & 60 & 5 & 216 & 10 & 432 & 560 & 750 & 5 y 10 \\
\hline A5 & $\mathrm{Al} / \mathrm{CaCO}_{3}$ & 17,7 & 60 & 5 & 216 & 20 & 432 & 580 & 720 & 6 \\
\hline A7 & $\mathrm{Al} / / \mathrm{CaCO}_{3} / \mathrm{Cu}^{*}$ & M & 10 & 10 & 194 & 15 & 194 & 300 & 640 & 7 \\
\hline A8 & $\begin{array}{c}\mathrm{Al} / \mathrm{NaHCO}_{3} \\
/ \mathrm{Cu}^{*}\end{array}$ & M & 20 & 10 & 195 & 20 & 195 & 150 & 900 & 2 \\
\hline A9 & $\mathrm{Al} * / \mathrm{NaHCO}_{3} / \mathrm{Cu} *$ & M & 20 & 10 & 195 & 20 & 195 & 150 & 900 & $\begin{array}{l}1 \mathrm{~min}, \\
30 \mathrm{~s}\end{array}$ \\
\hline $\mathrm{A} 12$ & $\mathrm{Al} / \mathrm{CaCO}_{3} / \mathrm{Cu}$ & M & 10 & 1 & 55,5 & 30 & 194 & 450 & 640 & 7 \\
\hline A13 & $\mathrm{Al} / \mathrm{CaCO}_{3} / \mathrm{Cu}^{*}$ & M & 10 & 1 & 55,5 & 30 & 194 & 450 & 640 & 7 \\
\hline
\end{tabular}

* Obtenido mecánicamente. M: Molienda Manual.

Tabla 1: Parámetros de muestras.

\subsection{Caracterización de las espumas}

La caracterización meso y microestructural de las espumas y los elementos constitutivos se estudiaron mediante microscopía óptica y tomografía de rayos x. La observación microscópica, previo tratamiento de desbaste y pulido, se realizó con el microscopio óptico marca Micoba M410 con Cámara digital Micoba 500, que nos permitió observar la muestras y determinar la necesidad de utilizar un método más complejo para determinar la calidad de las mismas.

Para las tomografías de rayos x se utilizó el microscopio de rayos x Xradia Micro XCT-200 disponible en la División Nuevos Materiales y Dispositivos (NuMaDi) del Centro Atómico Bariloche.

El desbaste se realizó con papel de desbaste de hasta 1000 mesh y el pulido con pasta de diamante de 9, 6, 3 y $1 \mu$ m en una pulidora metalográfica marca/modelo M P-1 Variable.

\section{Resultados y discusión}

\subsection{Análisis de los polvos por difracción de rayos $\mathrm{X}$}

Los resultados de los análisis de difracción de rayos $\mathrm{X}$ de los polvos de aluminio se observan en el gráfico 1. Se aprecia que en el polvo de aluminio comercial (Al en polvo en el gráfico) el grado de impurezas es mayor que en el aluminio obtenido mecánicamente y el Aluminio comercial del CAB (Centro Atómico Bariloche). 


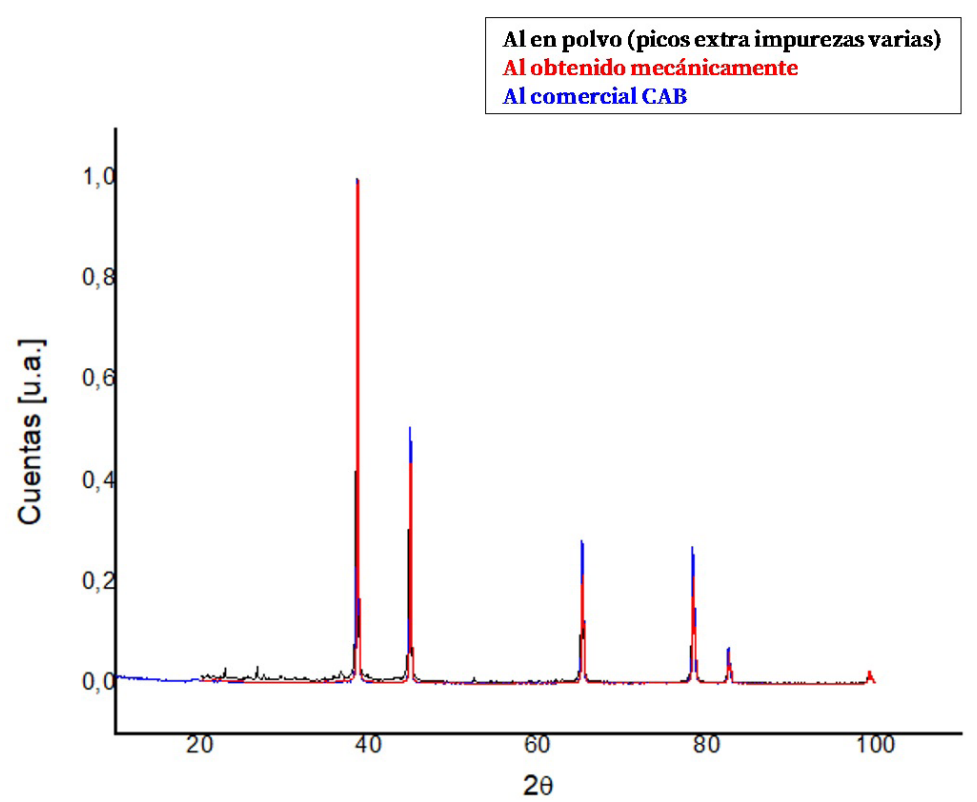

Gráfico 1: Comparativa de difracción de rayos $X$ de polvos de Al.

El número de picos adicionales del difractograma de nuestro aluminio comercial es un claro indicador de la existencia de impurezas indeseables en el polvo base.

Los resultados de los análisis de difracción de rayos X del polvo de cobre se observan en el gráfico 2. Se aprecia que el polvo de cobre obtenido mecánicamente contiene picos característicos del aluminio. En la comparativa con el polvo de cobre de CAB observamos el mismo patrón de difracción. El grado de contaminación de nuestro polvo obtenido mecánicamente puede deberse a una incorrecta manipulación y falta de limpieza de las herramientas de mecanizado.

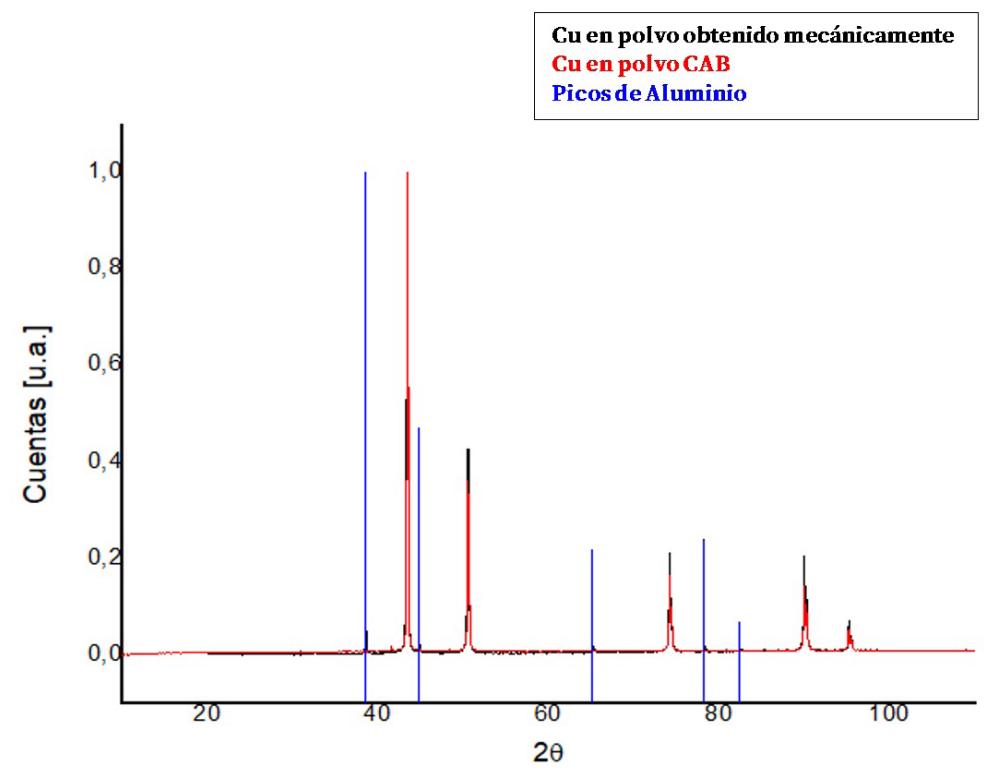

Gráfico 2: Comparativa de difracción de rayos $\mathrm{X}$ de polvos de $\mathrm{Cu}$. 


\subsection{Caracterización de espumas con polvo de aluminio comercial, polvo de cobre como aleante y $\mathrm{CaCO}_{3}$ cómo agente espumante.}

Dado que las muestras de la A1 a la A5 no fueron elaboradas con Cu cómo aleante, estas no se tuvieron en cuenta para la caracterización. Sólo las muestras A12 y A13 fueron objeto del estudio. Sin embargo, en la figura 1 puede observarse el estado de las muestras A1 a A5 luego del proceso final.
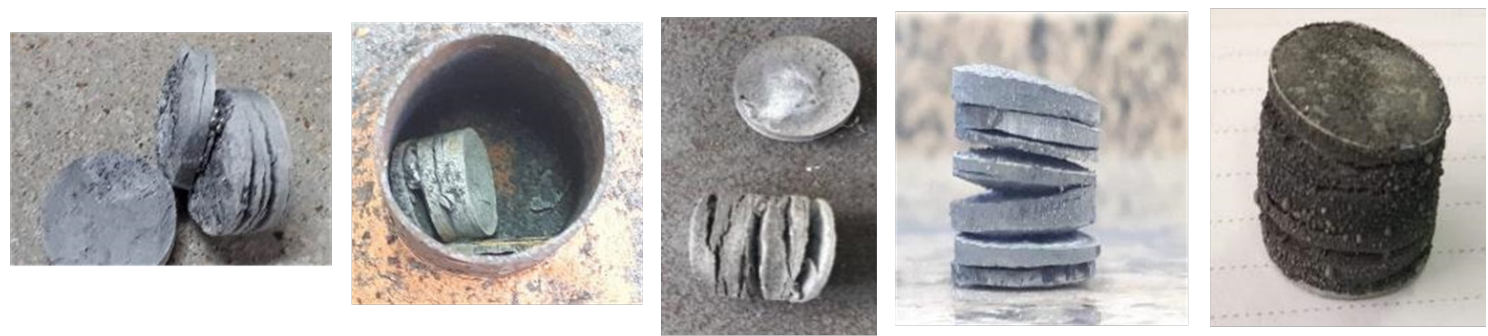

Fig.1: Muestras A1, A2, A3, A4 y A5.

En las figuras 2 y 3 se presentan las muestras A12 y A13 respectivamente, donde se observa el estado verde de las mismas, obtenidas por el mismo proceso.

La diferencia entre ambas radica en el polvo de cobre que en el caso de la muestra A12 se utilizó el comercial y en la A13 el obtenido mecánicamente, no así en las proporciones de la mezcla que para ambos casos se utilizó en un $11 \%$ en peso del metal aleante.

Los porcentajes de elementos aleantes se definen del diagrama de fases binario aluminio-cobre y se busca una zona en la que conviven dos estados, sólido y líquido a la temperatura de liberación del gas de los agentes espumantes de manera de darle estabilidad a la formación de la espuma.

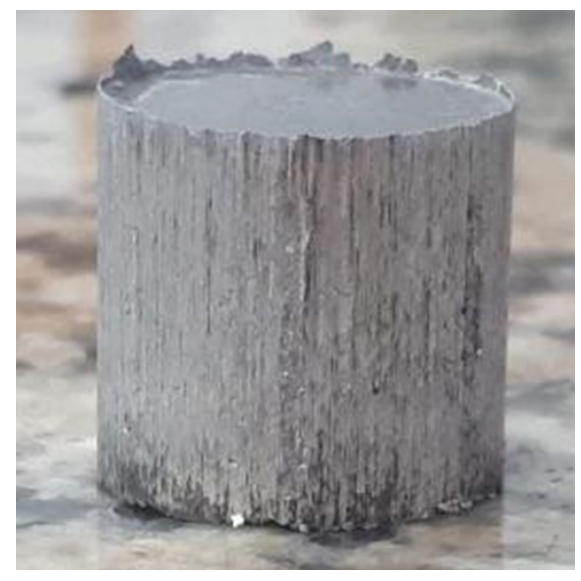

Fig. 2: Verde de muestra A12. 


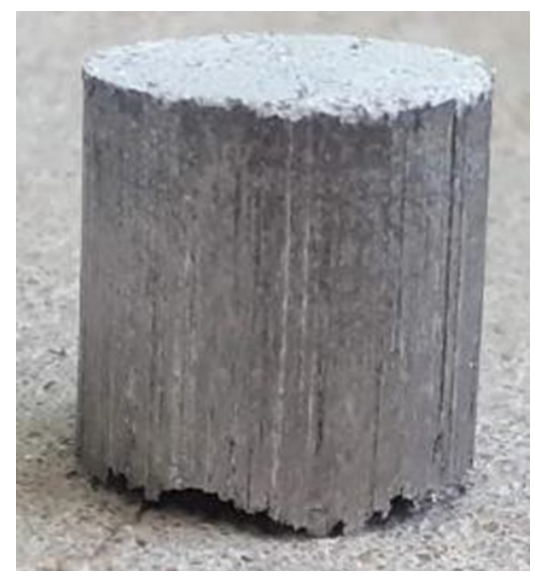

Fig. 3: Verde de muestra A13.

En las figuras 4 y 5 se observan las muestras luego del espumado, el cual se realizó a una temperatura de $640{ }^{\circ} \mathrm{C}$ por un período de tiempo de 7 minutos para ambas muestras.

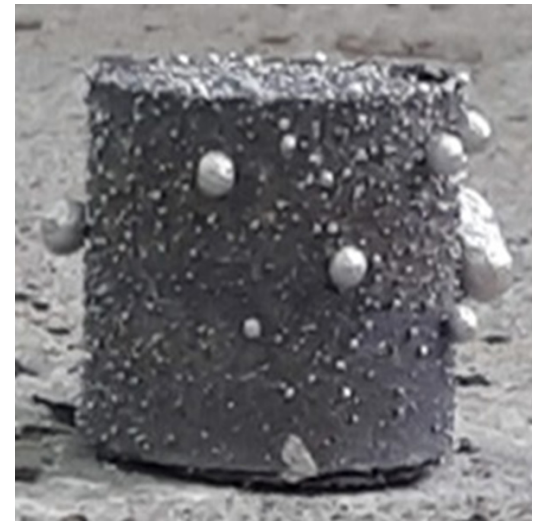

Fig. 4: Espuma de muestra A12.

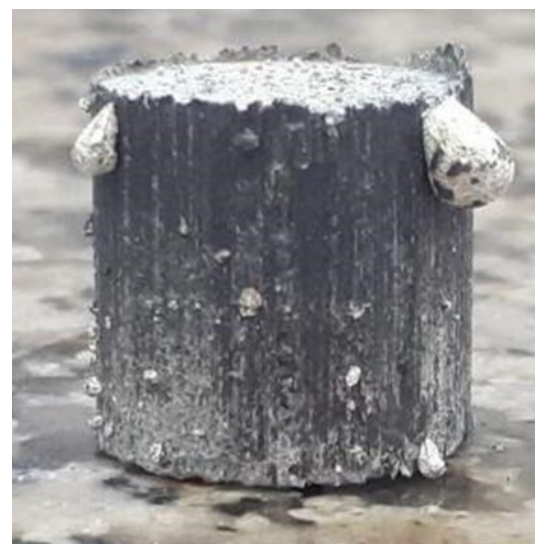

Fig. 5: Espuma de muestra A13. 
Se observa que se produjo liberación de gas del agente espumante debido al desplazamiento del material hacia el exterior, lo que revela que el proceso previo resultó con el grado de compactación requerido para el espumado.

Se determina que si bien existe una leve expansión en el espumado la misma no es homogénea en todo el volumen del material.

En las figuras 6 y 7 obtenidas del microscopio óptico se pueden ver porosidad remanente que por su tipología y por la forma exterior de las muestras previamente analizadas pueden deberse solamente a la falta de densificación del material en verde, lo cual no imposibilita el espumado de la muestra.

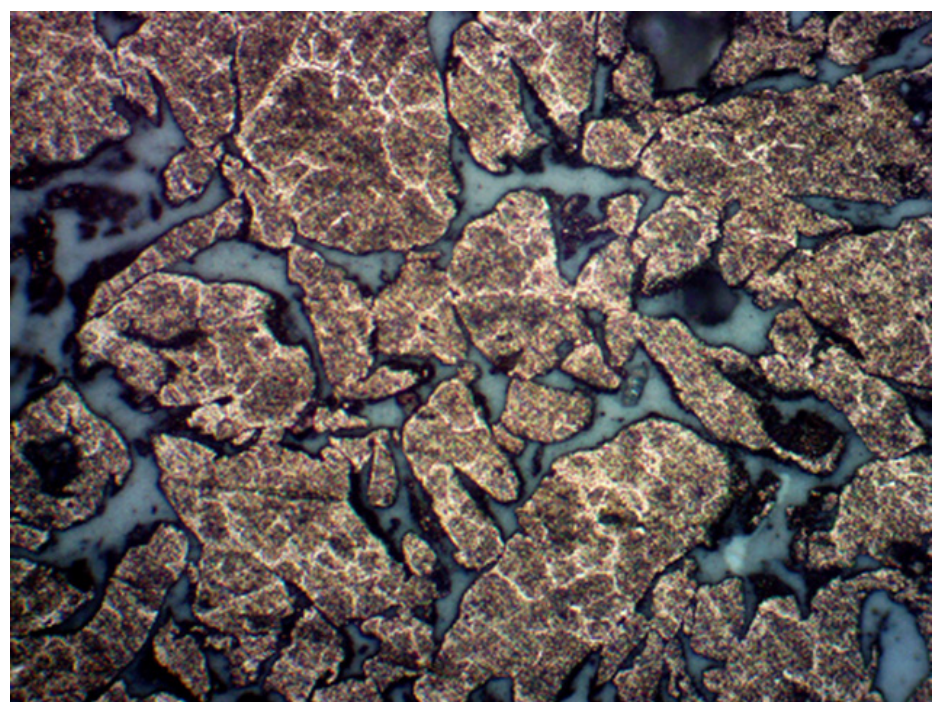

Fig. 6: 100 aumentos de la muestra A13, se observa la distancia por porosidad remanente entre las partículas de aluminio.

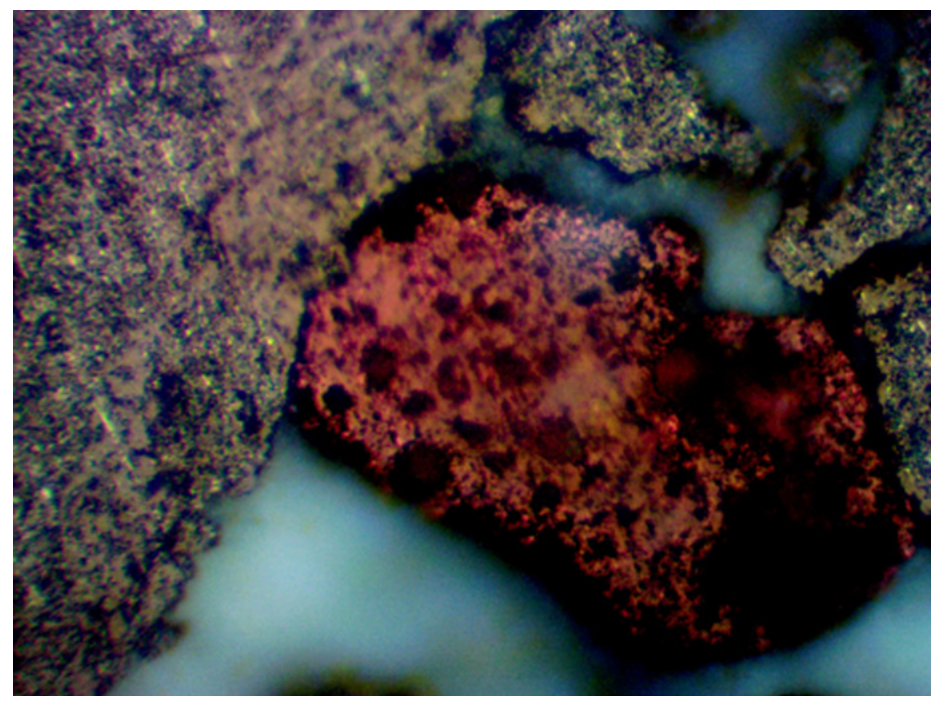

Fig. 7: 1000 aumentos. Distancia entre partículas de aluminio y cobre. 


\subsection{Caracterización de espumas con polvo de aluminio reciclado procesado mecánicamente, polvo de cobre como aditivo y cómo agentes espumantes $\mathrm{CaCO}_{3}$ y $\mathrm{NaHCO}_{3}$.}

En las figuras 8, 9 y 10 correspondiente a las muestras A7, A8 y A9 se observan en su estado verde posterior al retiro de la matriz, previo al espumado.

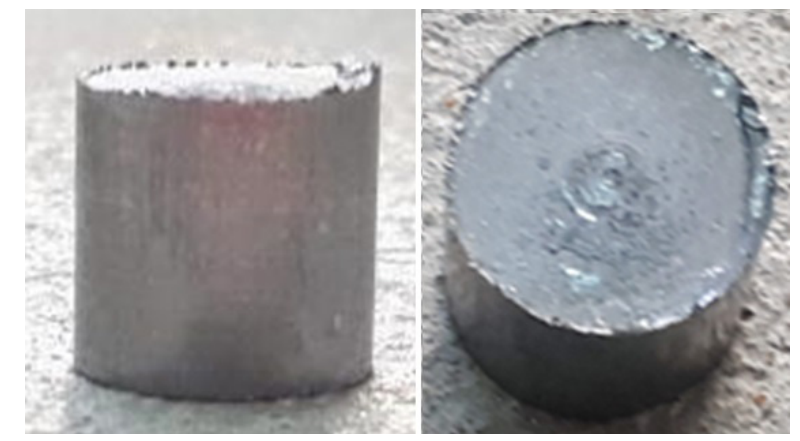

Fig. 8: muestra A7 en su estado verde. Vistas frontal y superior.

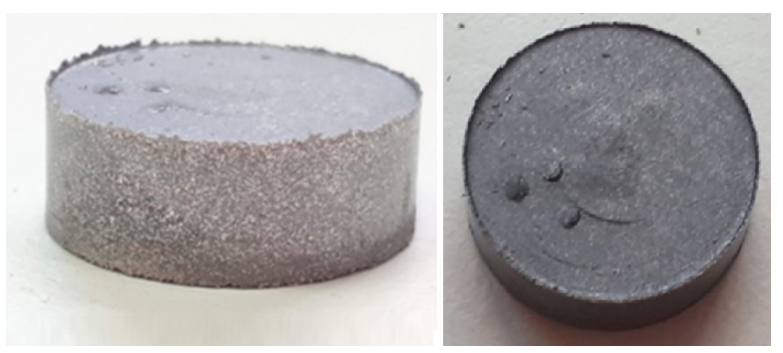

Fig. 9: muestra A8 en su estado verde. Vistas frontal y superior.

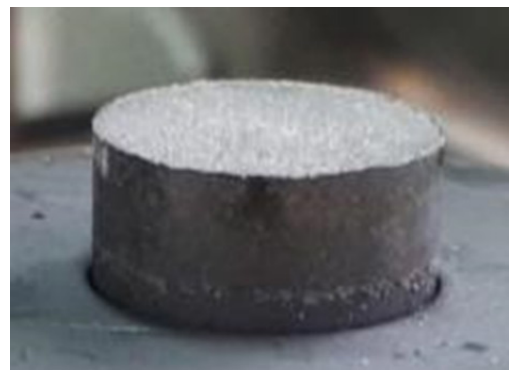

Fig. 10: muestra A9 en su estado verde. Vista frontal.

Como se detalló en el proceso experimental, se partió de partículas de aluminio reciclado que resultaron de mayor tamaño que el polvo comercial, verificando visualmente que dicho efecto podría no ser una influencia en la densificación de las muestras en verde.

Se observa que el uso de partículas de mayor tamaño al comercial favorece y mejora el proceso de prensado. La fricción entre partículas de polvo hace que no puedan deslizar 
entre ellas produciendo fallas de compactación. Esta fricción está dominada principalmente por el área superficial. De esta manera, cuanto menor sea el tamaño de partícula del polvo, mayor será el área superficial y por lo tanto mayor la fricción entre partículas, produciendo una compactación deficiente (Randall, 2005). Sin embargo, esta no es la única variable que determina el grado de densificación en su estado verde, ya que se pueden considerar además la forma y porosidad de las partículas, variación de su tamaño y la distribución dentro de la matriz de compactación entre otras.

Del proceso de espumado se observan las figuras 11, 12 y 13. La muestra A7 revela desplazamiento del material hacia el exterior, lo que evidencia la expansión de gas dentro de la muestra en el proceso de espumado. Sin embargo, no se observa un incremento apreciable respecto del volumen inicial de la muestra en su estado verde.

Las muestras A8 y A9 a diferencia de la A7, muestran, tanto desplazamiento de material como una leve expansión respecto de su estado verde, previo al espumado. Ambas presentan, a simple vista, una expansión y liberación superior del gas que las muestras donde se utiliza $\mathrm{CaCO}_{3}$ como agente espumante.

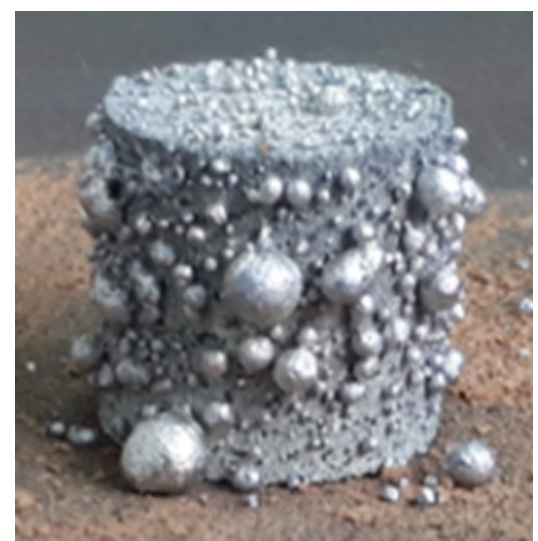

Fig. 11: Muestra A7

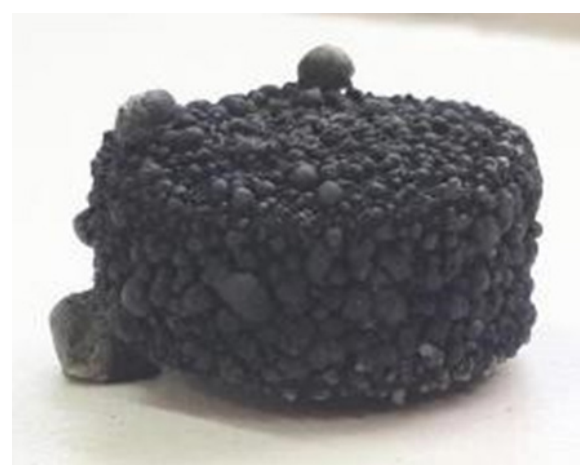

Fig. 12: Muestra A8. 


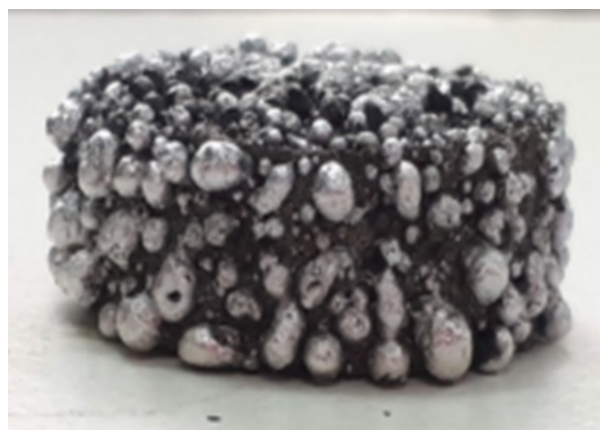

Fig. 13: Muestra A9.

Las muestras A7 y A9 se analizaron mediante tomografía de rayos $\mathrm{X}$ debido a que se encuentran, en conjunto con la A8, entre las espumas de mayor espumado y mejor homogeneidad. Los resultados obtenidos se observan en las imágenes siguientes.

En la figura 14, se observa un corte longitudinal de la muestra A7, en el cual se aprecia la presencia de poros distribuidos homogéneamente en su volumen. Se revela la existencia de grietas normales al sentido de compresión del proceso de prensado para la obtención del verde.
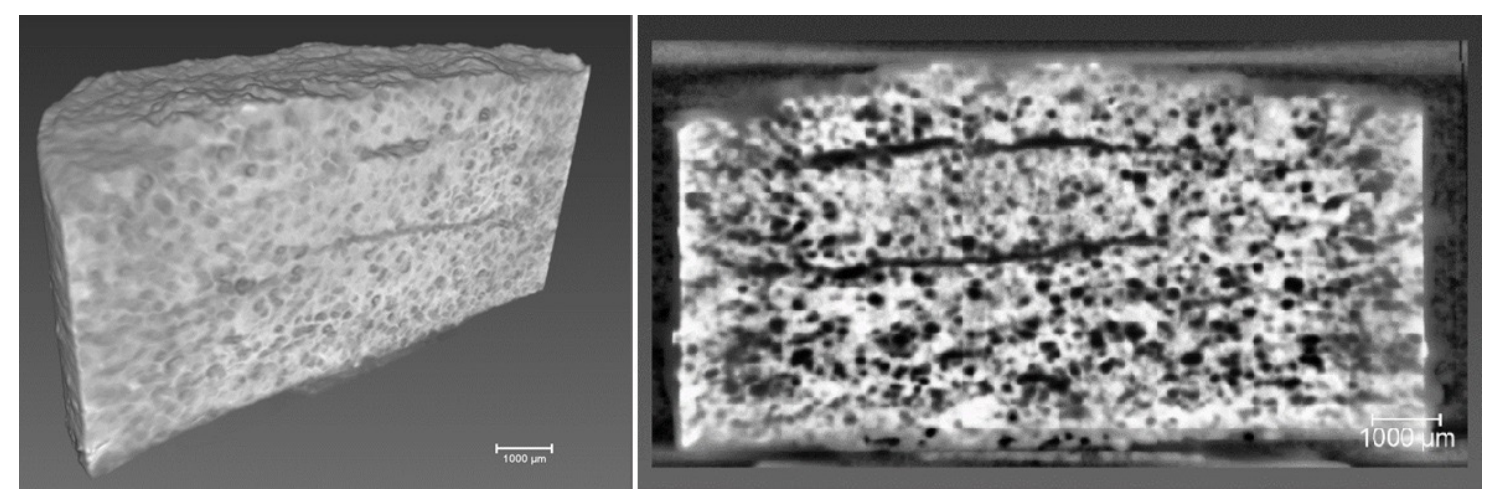

Fig. 14: muestra A7: a-Vista en perspectiva de un corte. b- Vista frontal de un corte.

En la figura 15 se realiza el análisis de los tres planos principales y como en la imagen anterior también se observan poros uniformemente distribuidos y la aparición de grietas transversales. 

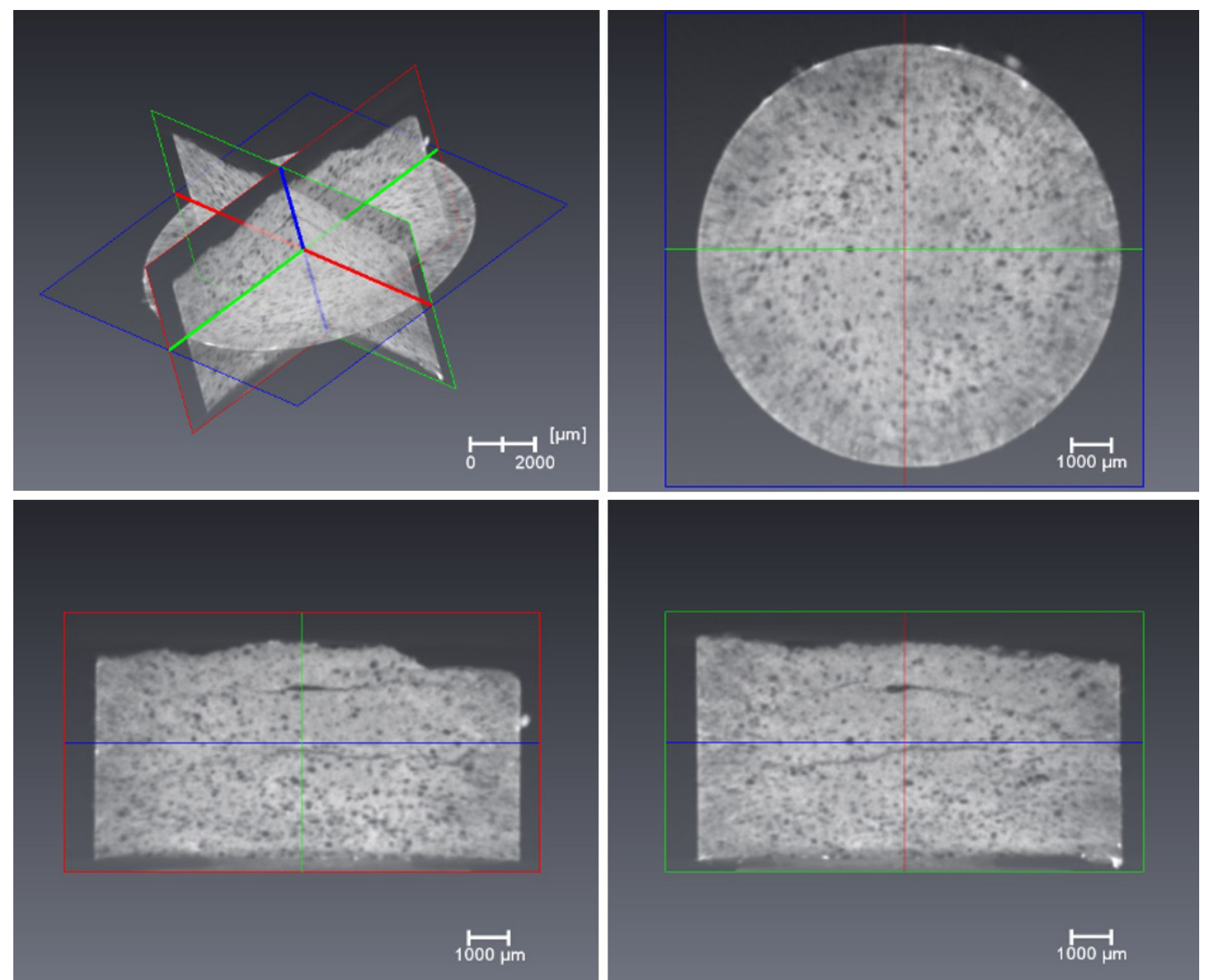

Fig. 15: muestra A7: a-Vista de los 3 planos. b- Vista de corte transversal plano XY. cVista de corte longitudinal plano XZ. d- Vista de corte longitudinal plano YZ.

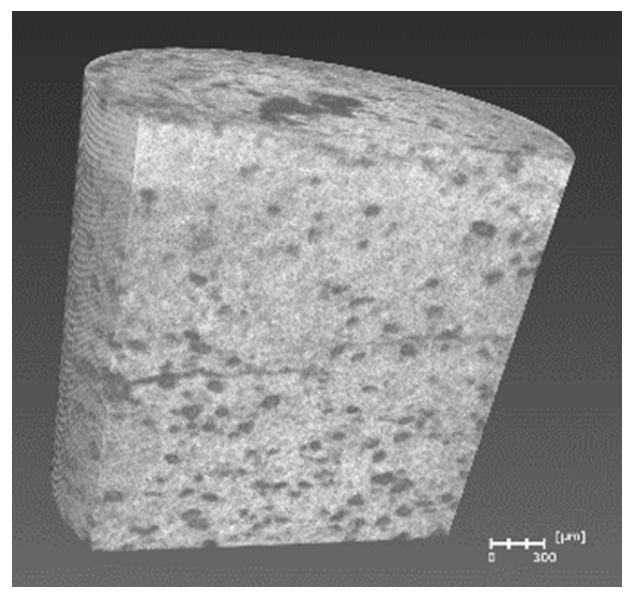

Fig. 16: corte volumétrico de la muestra A7.

En los planos XZ e YZ de la figura 15 como en el corte volumétrico del núcleo de la figura 
16, se observan grietas la que podrían deberse al escape del gas. Esto evidencia la expansión del material en la dirección $\mathrm{Z}$ que se corresponde con la dirección del prensado por lo que podría atribuirse esta falla a la falta de presión, temperatura o tiempo en el proceso previo al espumado (hotpressing). De la bibliografía consultada (Geramipour et al., 2017) se asume que la presión es correcta por lo que se propone, para mejorar la muestra aumentar la temperatura y/o tiempo de tratamiento. En esta última figura además se aprecia una densificación de poros no homogénea de ese volumen aislado.

En la figura 17a se observa la expansión en todas las direcciones de la muestra A9. En sus cortes transversales y longitudinales, figura $17 \mathrm{~b}$ y $17 \mathrm{c}$ respectivamente, no se aprecian fisuras en ninguna dirección, pero la complejidad de la superficie dificulta su visualización. Se aprecia un volumen poroso de aspecto visual no homogéneo con zonas que dejan pasar fácilmente los rayos X. La dificultad de visualización podría deberse al sodio residual en la muestra resultante de la descomposición del espumante.

En base a las muestras obtenidas empleando bicarbonato de sodio como espumante se propone variar la temperatura del espumado y el tiempo en el horno.
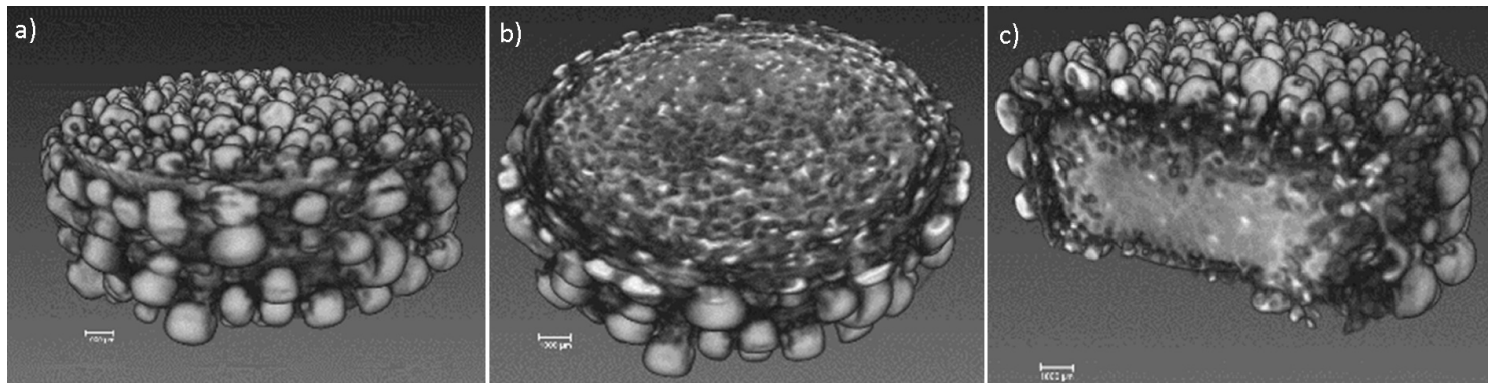

Fig. 17: muestra A9: a- Volumen completo. b- Vista corte transversal. c-Vista longitudinal.

En la figura 18, correspondiente a un corte volumétrico de la muestra A9, no se observan grietas normales a la dirección del prensado. Por lo que el aumento del tiempo en el proceso para la obtención del verde logra mejores resultados que el aumento de la temperatura. La aparición de poros no se hace presente en todo el volumen del material, mostrándose este como un continuo en determinadas zonas, produciéndose un gradiente de este a una fase de alta densidad de poros.

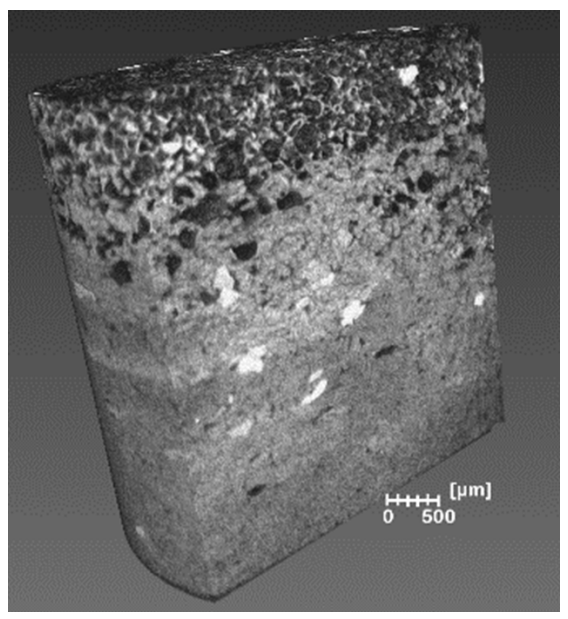

Fig. 18: corte volumétrico de la muestra A9. 


\section{Conclusiones}

- Los métodos experimentales utilizados en conjunto con los agentes espumantes alternativos para las aleaciones propuestas nos permitieron obtener espumas.

- Las muestras espumadas con bicarbonato de sodio evidencian expansión volumétrica en todas sus direcciones.

- En la obtención de muestras donde se empleó carbonato de calcio como agente espumante se evidencio visualmente una liberación del gas en dirección radial y longitudinal permitiendo la distribución homogénea de células.

- Las fisuras aparecen en los planos normales a la dirección de compresión ejercida para la obtención de la muestra en su estado verde. Esto puede deberse a la dirección de liberación del gas del agente espumante, la liberación del gas cuando el aluminio está todavía en estado sólido y/o a la posición en que se encuentra la muestra en el momento del espumado.

- Para el tamaño de las muestras estudiadas se verificó que la inercia térmica producida por la aleación aluminio-cobre dentro del horno, favoreció el espumado debido a que permitió obtener una viscosidad adecuada en el momento de la liberación del gas.

\section{Agradecimientos}

- A OUTOKUMPU FORTINOX SA por los cilindros de acero de alta resistencia.

- Taller Rojo y Verde por los trabajos de mecanizado. Villa Ángela, Provincia de Chaco.

- A BIOTEC: Grupo de Investigación en Biotecnología y Alimentos (UTN Frre). Directora: Dra. Carola Sosa. 


\section{Referencia}

Calcium carbonate. (s. f.). Sigma-Aldrich. Recuperado 15 de enero de 2022, de https://www. sigmaaldrich.com/AR/es/product/sigald/239216

Fernández, P., Cruz; L. y Coleto, J. (2009). Proceso de fabricación de metales celulares. Parte II: vía sólida, disposición de metales, otros procesos. Revista de Metalurgia de Madrid, 45, 124-142.

Gergely, V., Currand, D. y Clyne, T. (2003). The FOAMCARP process: Foaming of aluminium MMCs by the chalk-aluminium reaction in precursors [J]. Composites Science and Technology, 63, 2301-2310.

Geramipour,T. y Oveisi, H. (2017). Effects of foaming parameters on microstructure and compressive properties of aluminum foams produced by powder metallurgy method. ScienceDirect, 27, 1569-1579.

Kevorkijan, V., Skapin, S., Paulin, I., Sustarsic, B., Jenko, M. y Lazeta, M. (2011). Influence of the foaming precursor`s composition and density on the foaming efficiency, microstructure development and mechanical properties of aluminium foams. Original scientific, 45, 95-103.

Nakamura, T., Gnyloskurenko, S., Sakamoto, K., Byakova,A. y Ishikawa, R. (2002). Development of New Foaming Agent for Metal Foam. Materials Transactions, 43 (5), 1191 1196.

Byakova, A., Gnyloskurenko, S., y Nakamura, T. (2017). Effect of $\mathrm{CaCO}_{3}$ Foaming Agent at Formation and Stabilization of Al-Based Foams Fabricated by Powder Compact Technique. Materials Transactions, 58 (2), 249 - 258.

Bryant, J., Clowley, M., Wilhelmy, M., Kallivayalil, J. y Wang, W. (2008). In Metfoam DEStech Publications, Inc., Lancaster, PA, 2008, pp. 27.

Randall, G. (2005). Powder Metallurgy \& Particulate Materials Processing. Editor Metal Powder Industry, ISBN: 0-9762057-1-8

Sodium bicarbonate. (s. f.). Sigma-Aldrich. Recuperado 15 de enero de 2022, de https:// www.sigmaaldrich.com/AR/es/product/sial/s6297

Titanium (II) hydride. (s. f.). Sigma-Aldrich. Recuperado 15 de enero de 2022, de https:// www.sigmaaldrich.com/AR/es/product/sigald/209279 